\title{
Construction of Periodic Counterexamples to the Discrete-Time Kalman Conjecture
}

\author{
Peter Seiler and Joaquin Carrasco
}

\begin{abstract}
This paper considers the Lurye system of a discrete-time, linear time-invariant plant in negative feedback with a nonlinearity. Both monotone and slope-restricted nonlinearities are considered. The main result is a procedure to construct destabilizing nonlinearities for the Lurye system. If the plant satisfies a certain phase condition then a monotone nonlinearity can be constructed so that the Lurye system has a non-trivial periodic cycle. Several examples are provided to demonstrate the construction. This represents a contribution for absolute stability analysis since the constructed nonlinearity provides a less conservative upper bound than existing bounds in the literature.
\end{abstract}

\section{INTRODUCTION}

The discrete-time absolute stability problem considers the Lurye system of a discrete-time, linear time-invariant (LTI) plant in negative feedback with a nonlinearity. Let $k_{A S}$ denote the supremum of the set of values of $k$ for which the Lurye system is stable for all nonlinearities whose slope is restricted to $[0, k]$. It remains an open question to provide necessary and sufficient conditions to compute this maximal stability interval $k_{A S}$. The LTI Zames-Falb multipliers [1]-[6] provide a sufficient condition for stability. Specifically, the search over discrete-time Zames-Falb multipliers in [7] provides a lower bound $k_{Z F} \leq k_{A S}$. It has been conjectured in [8], [9] that this condition is actually necessary and sufficient, i.e. $k_{Z F}=k_{A S}$. In other words, the conjecture is that if a ZamesFalb multiplier does not exist for some $k$ then there exists a destabilizing nonlinearity whose slope remains within $[0, k]$.

The main contribution of this paper is a method to systematically construct destabilizing nonlinearities for the Lurye system. Such nonlinearities provide upper bounds $\bar{k} \geq k_{A S}$ and hence are complementary to the Zames-Falb conditions. The construction is based on a frequency-domain condition developed in [9] from the dual problem of the ZamesFalb condition. The construction is first described for Lurye systems with monotone nonlinearities (Section V-A). If the plant satisfies a phase condition at one frequency then there is a monotone nonlinearity such that the Lurye system has a non-trivial periodic solution. The destabilizing nonlinearity is explicitly constructed from the periodic solution. Next, the results are extended to Lurye systems with slope-restricted nonlinearities via a loop transformation (Section $\mathrm{V}$-B).

P. Seiler is with the Department of Electrical Engineering \& Computer Science, University of Michigan, Ann Arbor, US. pseilerdumich. edu

J. Carrasco is with the Department of Electrical \& Electronic Engineering, University of Manchester, M13 9PL, UK. joaquin.carrasco@manchester.ac.uk

This work was partially supported by EPSRC project EP/S03286X/1.
The only existing method to systematically construct a destabilizing nonlinearity is, to our knowledge, given by the Nyquist criterion. This provides the smallest linear gain, referred to as the Nyquist gain $k_{N}$, that destabilizes the Lurye system (Section VI). The Nyquist gain provides another upper bound $k_{N} \geq k_{A S}$ but it is known that this upper bound is conservative. Specifically, the discrete-time Kalman conjecture is that $k_{N}=k_{A S}$. This conjecture was shown to be false in [10], [11] and hence $k_{N}>k_{A S}$ in general. Our paper constructs destabilizing nonlinearities with slope restricted to $[0, \bar{k}]$. If $\bar{k}<k_{N}$ then the destabilizing nonlinearity represents a counterexample to the Kalman conjecture.

It is worth noting that the construction of counterexamples of the continuous-time Kalman Conjecture has been investigated since the sixties. It still attracts interest due to the illposed numerical issues [12]-[15]. For the Aizerman conjecture, a systematic analysis of the existence of periodic cycles for second-order systems has been explored in [16], [17]. In the context of optimization, construction of nonlinearities for worst-case convergence rate has been used in [18].

\section{NotATiON}

The set of integers and positive, natural numbers are denoted as $\mathbb{Z}$ and $\mathbb{N}^{+}$, respectively. $\mathbb{R} \mathbb{H}_{\infty}$ denotes the space of real, rational functions with all poles inside the open unit disk. This space corresponds to transfer functions for stable, LTI discrete-time systems. A function $\phi: \mathbb{R} \rightarrow \mathbb{R}$ has slope restricted to $[0, k]$ for some finite $k>0$ if:

$$
0 \leq \frac{\phi\left(y_{2}\right)-\phi\left(y_{1}\right)}{y_{2}-y_{1}} \leq k \quad \forall y_{2} \neq y_{1}
$$

$S_{0, k}$ with $k<\infty$ denotes the set of all functions with slope restricted to $[0, k]$ The notation $S_{0, k}$ with $k=\infty$ corresponds to the special case where $\phi$ is multivalued and monotone: $y_{2} \geq y_{1}$ implies $\phi\left(y_{2}\right) \geq \phi\left(y_{1}\right)$. In this case, $u \in \phi(y)$ will denote that $u$ is one of the values taken by $\phi$ at $y$. In addition, $S_{0, k}^{\text {odd }}$ denotes the set of all odd functions with slope restricted to $[0, k]$, i.e. $\phi(x)=-\phi(-x)$ for all $x \in \mathbb{R}$.

Finally, let $\left\{h_{0}, h_{1}, \ldots, h_{T-1}\right\}$ denote a finite sequence of real numbers. We will often stack such sequences into a column vector $H_{T}:=\left[h_{0}, h_{1}, \ldots, h_{T-1}\right]^{\top} \in \mathbb{R}^{T}$. The circulant matrix for a given finite sequence $H_{T}$ is defined as:

$$
C\left(H_{T}\right):=\left[\begin{array}{cccccc}
h_{0} & h_{T-1} & h_{T-2} & \cdots & h_{2} & h_{1} \\
h_{1} & h_{0} & h_{T-1} & \cdots & h_{3} & h_{2} \\
h_{2} & h_{1} & h_{0} & \cdots & h_{4} & h_{3} \\
\vdots & \vdots & \vdots & \ddots & \vdots & \vdots \\
h_{T-2} & h_{T-3} & h_{T-4} & \cdots & h_{0} & h_{T-1} \\
h_{T-1} & h_{T-2} & h_{T-3} & \cdots & h_{1} & h_{0}
\end{array}\right] .
$$




\section{PRoBlem STATEMENT}

Let $G$ be a discrete-time system that is LTI and singleinput, single-output (SISO). We consider the Lurye system of $G$ in negative feedback with a nonlinearity $\phi: \mathbb{R} \rightarrow \mathbb{R}$ as shown in Figure 1. The Lurye system is expressed as

$$
y=G u, \quad u_{k}=-\phi\left(y_{k}\right),
$$

We consider functions in $S_{0, k}$ with $k>0$ and $\phi(0)=0$. Lurye systems with both $k<\infty$ and $k=\infty$ will be considered. These cases are related by a loop transformation as discussed later in the paper. Additional details on this formulation can be found in [19].

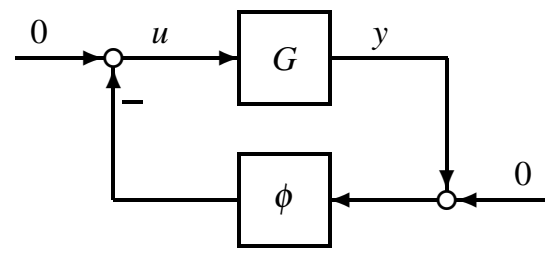

Fig. 1: Autonomous Lurye system

We provide conditions on $G$ for the existence of nontrivial periodic solutions to the Lurye system in Figure 1 . Specifically, let the plant $G$, slope constant $k>0$, and time horizon $T \in \mathbb{N}^{+}$be given. We provide sufficient conditions for the existence of a nonlinearity $\phi \in S_{0, k}$ with $\phi(0)=0$ such that the Lurye system has a non-trivial $T$-periodic solution. If the conditions are feasible then the proof provides a construction for the periodic signals $U_{T} \in \mathbb{R}^{T}$ and $Y_{T} \in \mathbb{R}^{T}$. A nonlinearity $\phi \in S_{0, k}$ can then be constructed to interpolate $\left(Y_{T},-U_{T}\right)$ and $(0,0)$.

\section{Preliminary Results}

This section presents two preliminary results that are used in the derivation of the main results.

Lemma 1: Let $T \in \mathbb{N}^{+}$be given. Then $\delta \in[-\pi, \pi]$ satisfies $|\delta| \leq \pi / T$ if and only if

$$
\operatorname{Re}\left\{e^{j \delta} e^{j\left(\frac{\pi}{T} k+\frac{\pi}{2}\right)}\right\} \operatorname{Re}\left\{e^{j\left(\frac{\pi}{T} k+\frac{\pi}{2}\right)}\right\} \geq 0, \quad \text { for } k \in \mathbb{Z} .
$$

Proof: The result is trivially true for the case $T=1$, hence the rest of the proof considers the case $T \geq 2$. To simplify notation, define $z_{k}:=e^{j\left(\frac{\pi}{T} k+\frac{\pi}{2}\right)} \in \mathbb{C}$. The sequence $z_{k}$ has period $2 T$ with $z_{0}=0$ and $z_{k}=-z_{k+T}$. Thus Equation 4 is equivalent to:

$$
\operatorname{Re}\left\{e^{j \delta} z_{k}\right\} \operatorname{Re}\left\{z_{k}\right\} \geq 0 \quad \text { for } k=1,2, \ldots, T-1
$$

The phase of $\left\{z_{k}\right\}_{k=1}^{T-1}$ ranges from $\pi / 2+\pi / T$ up to $3 \pi / 2-$ $\pi / T$. Hence all values of $\left\{z_{k}\right\}_{k=1}^{T-1}$ have strictly negative real part. It follows that Equation 5 is equivalent to: $\operatorname{Re}\left\{e^{j \delta} z_{k}\right\} \leq$ 0 for $k=1,2, \ldots, T-1$. This can be written as the following inequality on the phase:

$$
\frac{\pi}{2} \leq \frac{\pi}{T} k+\frac{\pi}{2}+\delta \leq \frac{3 \pi}{2} \text { for } k=1,2, \ldots, T-1
$$

Thus Equation 4 holds if and only if (restricting $\delta \in[-\pi, \pi]$ ):

$$
-\frac{\pi}{T} k \leq \delta \leq \pi-\frac{\pi}{T} k \quad \text { for } k=1,2, \ldots, T-1
$$

This condition is equivalent to $|\delta| \leq \pi / T$.

The next result provides a necessary and sufficient condition to interpolate finite sequences by a multi-valued function in $S_{0, \infty}$. This result appears in Section 8 of [20] and more general finite interpolation results appear in [21] and [22].

Lemma 2 ([20]): Let finite sequences $\left\{y_{i}\right\}_{i=0}^{T-1}$ and $\left\{u_{i}\right\}_{i=0}^{T-1}$ be given. There exists $\phi \in S_{0, \infty}$ such that $-u_{i} \in \phi\left(y_{i}\right)$ for $i=0, \ldots, T-1$ if and only if:

$$
\left(y_{i}-y_{l}\right)\left(u_{i}-u_{l}\right) \leq 0 \quad \forall i, l \in\{0, \ldots, T-1\}
$$

A formal proof is given in [20]. If the finite sequences satisfy Equation 8 then there is, in general, more than one $\phi \in S_{0, \infty}$ that interpolates the data. Here we will provide an explicit formula for a $\phi \in S_{0, \infty}$ that interpolates the data. First, re-order the points so that $y_{0} \leq y_{1} \leq \cdots \leq y_{T-1}$ and $-u_{0} \leq$ $-u_{1} \leq \cdots \leq-u_{T-1}$. This re-ordering is possible since the data satisfy Equation 8 Next note that there can be repeats in the input data: $y_{i}=y_{i+1}=\cdots=y_{i+r}$ for some $r>0$. In this case the nonlinearity $\phi$ is multi-valued: $\phi\left(y_{i}\right) \in\left[-u_{i},-u_{i+r}\right]$. Finally, the re-ordered sequences are interpolated by the following multi-valued function:

$$
\phi(y) \subseteq \begin{cases}-u_{0} & \text { if } y<y_{0} \\ {\left[-u_{i},-u_{i+r}\right]} & \text { if } y=y_{i}=\cdots=y_{i+r} \\ & \text { for some } r \geq 0 \\ \left(f_{i}-1\right) u_{i}-f_{i} u_{i+1} & \text { if } y_{i}<y<y_{i+1} \\ & \text { where } f_{i}:=\frac{y-y_{i}}{y_{i+1}-y_{i}} \\ -u_{T-1} & \text { if } y>y_{T-1}\end{cases}
$$

This corresponds to linear interpolation or multi-valued output for any input $y \in\left[y_{0}, y_{T-1}\right]$ and nearest neighbor extrapolation otherwise. This specific nonlinearity has the following useful property:

Lemma 3: Suppose the finite sequences $\left\{y_{i}\right\}_{i=0}^{T-1}$ and $\left\{u_{i}\right\}_{i=0}^{T-1}$ are odd, i.e. $\left(y_{i}, u_{i}\right)$ is in the sequence if and only if $\left(-y_{i},-u_{i}\right)$ is in the sequence. Then the nonlinearity $\phi$ in Equation 9 is odd and has $0 \in \phi(0)$.

Proof: The proof is straightforward by construction of $\phi$ in Equation 9

\section{MAin Results}

\section{A. Construction for $S_{0, \infty}$}

Theorem 1 below provides conditions for the existence of $\phi \in S_{0, \infty}$ such that the Lurye system has a non-trivial $T$-periodic solution. The proof relies on the response of the LTI system $G \in \mathbb{R H}_{\infty}$ due to periodic inputs. Let $g:=$ $\left\{g_{0}, g_{1}, g_{2}, \ldots\right\}$ denote the impulse response of $G$. The convolution summation for a (not necessarily periodic) input sequence $\left\{u_{i}\right\}_{i=-\infty}^{\infty}$ is:

$$
y_{k}=\sum_{i=-\infty}^{k} g_{k-i} u_{i}
$$

Next, consider the case where the input is $T$-periodic so that $u_{i+T}=u_{i}$ for all $i$. The terms in convolution summation can be re-grouped. This yields the following $T$-periodic output

$$
y_{k}=\sum_{i=0}^{T} h_{k-i} u_{i} \text { where } h_{i}:=\sum_{l=0}^{\infty} g_{i+l T} .
$$


To simplify the notation, define the column vector $H_{T}:=$ $\left[\begin{array}{llll}h_{0} & h_{1} & \ldots & h_{T-1}\end{array}\right]^{\top} \in \mathbb{R}^{T}$. Similarly, stack the $T$-periodic sequences $\left\{u_{i}\right\}_{i=0}^{T-1}$ and $\left\{y_{i}\right\}_{i=0}^{T-1}$ into vectors $U_{T}$ and $Y_{T}$, respectively. The $T$-periodic inputs and outputs are related by $Y_{T}=C\left(H_{T}\right) U_{T}$ where $C\left(H_{T}\right)$ is the circulant matrix in Equation [2] We are now ready to state the main results.

Theorem 1: Let $G \in \mathbb{R H}_{\infty}$ and integers $0<\alpha<\beta$ be given. Assume $\alpha$ and $\beta$ are co-prime, i.e. their greatest common divisor is 1 . Define the frequency $\omega:=\frac{\alpha \pi}{\beta}$ with corresponding period $T=2 \beta$ if $\alpha$ is odd and $T=\beta$ if $\alpha$ is even. There exists $\phi \in S_{0, \infty}$ such that the Lurye system has a non-trivial $T$-periodic solution if

$$
\pi-\frac{\pi}{T} \leq \angle G\left(e^{j \omega}\right) \leq \pi+\frac{\pi}{T}
$$

where:

Proof: Define the $T$-periodic input $U_{T}:=\operatorname{Re}\left\{V_{T}\right\}$

$$
V_{T}:=\left[\begin{array}{llll}
1 & e^{j \omega} & \ldots & e^{j \omega(T-1)}
\end{array}\right] \in \mathbb{C}^{T} .
$$

Note that $V_{T}$ is an eigenvector of $C\left(H_{T}\right)$ with eigenvalue $G\left(e^{j \omega}\right)$ [23], [24]. Hence $C\left(H_{T}\right) V_{T}=G\left(e^{j \omega}\right) V_{T}$ and the $T$ periodic output is $Y_{T}=\operatorname{Re}\left\{C\left(H_{T}\right) V_{T}\right\}=\operatorname{Re}\left\{G\left(e^{j \omega}\right) V_{T}\right\}$.

Next, we show that the input/output sequences can be interpolated by a nonlinearity $\phi \in S_{0, \infty}$. If Equation 12 holds then $G\left(e^{j \omega}\right)=-r e^{j \delta}$ for some $r>0$ and $|\delta| \leq \pi / T$. Use the expressions for $U_{T}, Y_{T}$, and $G\left(e^{j \omega}\right)$ to show the following:

$\left(y_{i}-y_{l}\right)\left(u_{i}-u_{l}\right)=\operatorname{Re}\left\{-r e^{j \delta}\left(e^{j \omega i}-e^{j \omega l}\right)\right\} \operatorname{Re}\left\{e^{j \omega i}-e^{j \omega l}\right\}$.

The following identity holds for any integers $i$ and $l$ :

$$
e^{j \omega i}-e^{j \omega l}=2 \sin \left(\frac{\omega}{2}(i-l)\right) e^{j\left(\frac{\omega}{2}(i+l)+\frac{\pi}{2}\right)} .
$$

This identity yields:

$$
\left(y_{i}-y_{l}\right)\left(u_{i}-u_{l}\right)=-c \operatorname{Re}\left\{e^{j \delta} e^{j\left(\frac{\omega}{2}(i+l)+\frac{\pi}{2}\right)}\right\} \operatorname{Re}\left\{e^{j\left(\frac{\omega}{2}(i+l)+\frac{\pi}{2}\right)}\right\}
$$

where $c:=4 r \sin ^{2}\left(\frac{\omega}{2}(i-l)\right) \geq 0$. Finally, $\frac{\omega}{2}=\frac{\alpha \pi}{T}$ if $\alpha$ is odd or $\frac{\omega}{2}=\frac{\alpha \pi}{2 T}$ if $\alpha$ is even. In either case, $\frac{\omega}{2}(i+l)=\frac{\pi}{T} k$ for some integer $k$. It follows from Lemma 1 that $\left(y_{i}-y_{l}\right)\left(u_{i}-u_{l}\right) \leq 0$ for any $i, l \in\{0, \ldots, T-1\}$. By Lemma 2 , there exists $\phi \in$ $S_{0, \infty}$ such that $-u_{i} \in \phi\left(y_{i}\right)$ for $i=0, \ldots, T-1$.

The only remaining issue is to show that the multi-valued function satisfies $0 \in \phi(0)$. There are two cases:

A) $\alpha$ is odd: The frequency is $\omega=\frac{2 \pi \alpha}{T}$ where $T=2 \beta$ is even. The points in $V_{T} \in \mathbb{C}^{T}$ : (i) are equidistantly spaced around the unit circle, (ii) are symmetric about both the real and imaginary axis, (iii) and there is a rotational symmetry of $\pi$. The points in $C\left(H_{T}\right) V_{T}=G\left(e^{j \omega}\right) V_{T}$ are scaled and rotated by the magnitude and phase of $G\left(e^{j \omega}\right)$. If $G$ satisfies the phase constraint in (12) then these points are: (i) equidistantly spaced around a circle, (ii) they are rotated an angle $\delta$ with respect to $V_{T}$, (iii) and there is a rotational symmetry of $\pi$. As a result the interpolating data is odd: if $\left(y_{i},-u_{i}\right)$ is a point in the input/output data then $\left(-y_{i}, u_{i}\right)$ is as well. By Lemma 3 , the interpolating nonlinearity is not only monotone but is also odd and satisfies $0 \in \phi(0)$.

B) $\alpha$ is even: The frequency is $\omega=\frac{\alpha \pi}{T}$ where $T=\beta$ is odd. The points in $V_{T} \in \mathbb{C}^{T}$ are again equidistantly spaced around the unit circle and symmetric about the real axis.
However, the rotational symmetry of $\pi$ no longer holds and hence the sequence of points is not odd. As a result, the interpolated function is not odd. This is an expected property from the analysis in [9] for the case where $\alpha$ is even. More importantly, the interpolated function fails to satisfy $0 \in \phi(0)$. It is possible to shift the nonlinearity to recover $0 \in \phi(0)$. First, modify the definition of the input sequence to be $\hat{U}_{T}=\operatorname{Re}\left\{V_{T}\right\}+\xi \mathbf{1}$ where $\mathbf{1} \in \mathbb{R}^{T}$ is a vector of ones and $\xi$ is to be chosen. Note that $C\left(H_{T}\right) \mathbf{1}=G(1) \mathbf{1}$ where $G(1)=\sum_{k=0}^{\infty} g_{k}$ is the DC gain of the system. Thus the modified output sequence is:

$$
\hat{Y}_{T}=\operatorname{Re}\left\{C\left(H_{T}\right) \hat{U}_{T}\right\}=\operatorname{Re}\left\{G\left(e^{j \omega}\right) V_{T}\right\}+\xi G(1) \mathbf{1}
$$

This modification adds the constants $\xi$ and $\xi G(1)$ to the input and output sequences, respectively. The choice of $\xi$ shifts the original curve generated by $\left(Y_{T},-U_{T}\right)$ along the line connecting $(0,0)$ and $(G(1),-1)$. Find the intersection of the original curve with the line connecting $(0,0)$ and $(G(1),-1)$. This yields the value of $\xi$ so that the modified function satisfies $0 \in \phi(0)$. This function is, in general, nonodd and generates a $T$-periodic solution to the Lurye system.

If we restrict our attention to odd nonlinearities, i.e. $\phi \in S_{0, \infty}^{\text {odd }}$, the phase condition must be modified as follows:

Theorem 2: Let $G \in \mathbb{R H}_{\infty}$ and integers $0<\alpha<\beta$ be given. Assume $\alpha$ and $\beta$ are co-prime. Define the frequency $\omega:=\frac{\alpha \pi}{\beta}$ with corresponding period $T=2 \beta$ if $\alpha$ is odd and $T=\beta$ if $\alpha$ is even. There exists $\phi \in S_{0, \infty}^{\text {odd }}$ such that the Lurye system has a non-trivial $T$-periodic solution if

$$
\pi-\frac{\pi}{2 \beta} \leq \angle G\left(e^{j \omega}\right) \leq \pi+\frac{\pi}{2 \beta} .
$$

Proof: The statement with $\alpha$ odd follows from the proof of Theorem 1 If $\alpha$ is even then use the method in the proof of Theorem 1 to construct sequences $\left\{u_{i}\right\}_{i=0}^{\beta-1}$ and $\left\{y_{i}\right\}_{i=0}^{\beta-1}$. Next, append the data to include both $\left(y_{i},-u_{i}\right)$ and $\left(-y_{i}, u_{i}\right)$ for $i=0, \ldots, \beta-1$. The phase condition in (15) can be used to show that the appended data satisfies Equation 8 . Hence the data can be interpolated by a monotone nonlinearity $\phi$ (Lemma 2). Moreover, the appended data is odd and hence $\phi \in S_{0, \infty}^{\text {odd }}$ (Lemma 3). The appended data is only used in the function interpolation and the Lurye system will have a $\beta$ periodic solution with only $\left\{\left(y_{i},-u_{i}\right)\right\}_{i=0}^{\beta-1}$.

\section{B. Construction for $S_{0, k}$ with $k<\infty$}

Consider a Lurye system of $(G, \phi)$ where $\phi$ is slope restricted with $k<\infty$. The loop transformation in Figure 2 maps to a Lurye system $(\tilde{G}, \tilde{\phi})$ where $\tilde{\phi}$ is monotone.

Lemma 4: The Lurye system with $G \in \mathbb{R H}_{\infty}$ and $\phi \in S_{0, k}$ $\left(\phi \in S_{0, k}^{\text {odd }}\right)$ has a periodic solution if and only if the Lurye system with $\tilde{G}:=G+1 / k$ and $\tilde{\phi} \in S_{0, \infty}\left(\tilde{\phi} \in S_{0, \infty}^{\text {odd }}\right)$ has a periodic solution.

Proof: The proof follows from standard loop transformation arguments, see Chapter III, Section 6, in [19].

Proposition 1: Let $G \in \mathbb{R H}_{\infty}$ and integers $0<\alpha<\beta$ be given. Assume $\alpha$ and $\beta$ are co-prime. Define the frequency 


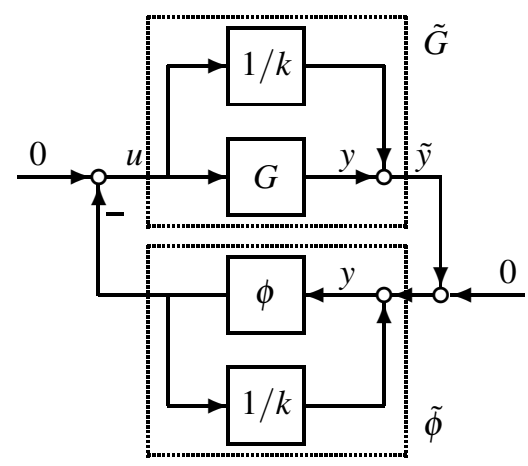

Fig. 2: Loop transformation for a Lurye systems

$\omega:=\frac{\alpha \pi}{\beta}$ with corresponding period $T=2 \beta$ if $\alpha$ is odd and $T=\beta$ if $\alpha$ is even. There is $\phi \in S_{0, k}$ with $k<\infty$ such that the Lurye system has a non-trivial $T$-periodic solution if

$$
R(\omega)+\frac{1}{k}<0 \text { and } \frac{-|I(\omega)|}{R(\omega)+1 / k} \leq \tan \left(\frac{\pi}{T}\right),
$$

where $R(\omega)=\operatorname{Re}\left\{G\left(e^{j \omega}\right)\right\}$ and $I(\omega)=\operatorname{Im}\left\{G\left(e^{j \omega}\right)\right\}$.

Proof: If (16) holds then $\tilde{G}:=G+1 / k$ satisfies the phase conditon in (12). By Theorem 1, there is a $\tilde{\phi} \in S_{0, \infty}$ such that the Lurye system of $(\tilde{G}, \tilde{\phi})$ has a non-trivial solution. This implies, by Lemma 4 that there is a $\phi \in S_{0, k}$ such that the Lurye system of $(G, \phi)$ has a non-trivial solution.

The destabilizing nonlinearity with the smallest slope bound $\bar{k}$ is obtained when the second constraint in (16) holds with equality. Solving this equality for $\bar{k}$ yields:

$$
\bar{k}=\frac{-\tan \left(\frac{\pi}{T}\right)}{R(\omega) \tan \left(\frac{\pi}{T}\right)+|I(\omega)|}
$$

If $G$ itself satisfies the phase condition in (12) then $\bar{k} \geq 0$. If $\bar{k}<0$ then no destabilizing nonlinearity exists. Finally, let $\left\{\tilde{y}_{i},-u_{i}\right\}_{i=0}^{T-1}$ be the data interpolated by $\tilde{\phi}$. The nonlinearity $\phi$ is obtained, after loop transforming back, by interpolating $\left\{\tilde{y}_{i}-u_{i} / k,-u_{i}\right\}_{i=0}^{T-1}$. The nonlinearity $\phi$ is no longer multivalued after the loop transformation.

Proposition 2: Let $G \in \mathbb{R H}_{\infty}$ and integers $0<\alpha<\beta$ be given. Assume $\alpha$ and $\beta$ are co-prime. Define the frequency $\omega:=\frac{\alpha \pi}{\beta}$ with corresponding period $T=2 \beta$ if $\alpha$ is odd and $T=\beta$ if $\alpha$ is even. There is $\phi \in S_{0, k}^{\text {odd }}$ with $k<\infty$ such that the Lurye system has a non-trivial $T$-periodic solution if

$$
R(\omega)+\frac{1}{k}<0 \text { and } \frac{-|I(\omega)|}{R(\omega)+1 / k} \leq \tan \left(\frac{\pi}{2 \beta}\right)
$$

where $R(\omega)=\operatorname{Re}\left\{G\left(e^{j \omega}\right)\right\}$ and $I(\omega)=\operatorname{Im}\left\{G\left(e^{j \omega}\right)\right\}$.

The proof is similar to that given for Proposition 1 and is omitted. Moreover, we can solve for the smallest $\bar{k}^{\text {odd }}$ for which there is a destabilizing $\phi \in S_{0, k}^{\text {odd }}$.

\section{Discussion on the Kalman Conjecture}

The constructed nonlinearity is valid for each $(\alpha, \beta)$ where the phase condition is satisfied at the frequency $\omega=\frac{\alpha \pi}{\beta}$. This provides an upper bound $\bar{k}$ on the stability boundary $k_{A S}$ for the absolute stability problem. The Nyquist gain provides an alternative upper bound using the class of linear gains.
Definition 1 (Nyquist gain): The Nyquist gain of $G \in$ $\mathbb{R H}_{\infty}$, denoted $k_{N}$, is the supremum of the set of gains $k$ such that the feedback interconnection between $G$ and $K$ is stable for all $K \in[0, k]$.

The constructed nonlinearity only provides new information if $\bar{k}<k_{N}$. To clarify further, recall the Discrete-Time Kalman Conjecture (DTKC) is that $k_{N}=k_{A S}$ as stated next.

Conjecture 1 (DTKC [10], [25]): The Lurye system with $G$ and any $\phi \in S_{0, k}$ is stable if and only $k<k_{N}$.

Our nonlinear construction does not provide any valuable information beyond the Nyquist value for plants where $k_{Z F} \simeq k_{N}$. However, as DTKC is false in general [11], the Nyquist gain is a conservative upper bound. Our construction becomes relevant for the plants used in absolute stability literature such as the examples in [7], where there is a significant gap between $k_{Z F}$ and $k_{N}$ (see Tables I and II in [9]). For all the six examples in [9], our construction leads to counterexamples of the DTKC, i.e. $\bar{k}<k_{N}$ (see Table III in [9]).

\section{NUMERICAL EXAMPLES}

\section{A. Example with $\alpha$ odd and $k=\infty$}

To illustrate the main results, first consider artificially constructed plants. Let $\alpha=1$ and $\beta=5$ so that $\omega=\pi / 5$. The periodic solutions have period $T=10$. Consider a plant $G_{1}$ with $G_{1}\left(e^{j \omega}\right)=-e^{j \frac{\pi}{25}}$. This plant satisfies the phase condition in Equation 12 of Theorem 1 The input and output of $G_{1}$ are $U_{T}=\operatorname{Re}\left\{V_{T}\right\}$ and $Y_{T}=\operatorname{Re}\left\{G_{1}\left(e^{j \omega}\right) V_{T}\right\}$ where:

$$
\begin{aligned}
V_{T} & :=\left[\begin{array}{llllllll}
1 & e^{j \frac{\pi}{5}} & e^{j \frac{2 \pi}{5}} & e^{j \frac{3 \pi}{5}} & \cdots & e^{j \frac{7 \pi}{5}} & e^{j \frac{8 \pi}{5}} & e^{j \frac{9 \pi}{5}}
\end{array}\right]^{\top} \\
& :=\left[\begin{array}{llllllll}
1 & e^{j \frac{\pi}{5}} & e^{j \frac{2 \pi}{5}} & e^{j \frac{3 \pi}{5}} & \cdots & e^{-j \frac{3 \pi}{5}} & e^{-j \frac{2 \pi}{5}} & e^{-j \frac{\pi}{5}}
\end{array}\right]^{\top}
\end{aligned}
$$

Figure 3 plots the vectors $V_{T}$ (red) and $G_{1}\left(e^{j \omega}\right) V_{T}$ (blue) in the complex plane. The projection of these points onto the real axis corresponds with the input-output data $U_{T}$ and $Y_{T}$. In this example $\alpha$ is odd. Note that the points in $V_{T}$ (i) are equidistantly spaced around the unit circle, (ii) are symmetric about both the real and imaginary axis, (iii) and there is a rotational symmetry of $\pi$. These are the key properties claimed in Theorem 1 The points in $G_{1}\left(e^{j \omega}\right) V_{T}$ are shifted slightly counterclockwise. Figure 4 shows the interpolated function (blue) obtained from $\left(Y_{T},-U_{T}\right)$ using Equation 9 This function is odd and passes through $\phi(0)=0$.

Next consider a plant $G_{2}$ with $G_{2}\left(e^{j \omega}\right)=-e^{j \frac{\pi}{10}}$ and the same $(\alpha, \beta, \omega)$ as given above. This plant satisfies the phase condition in Equation 12 but with equality, i.e. the phase condition is tight. The input and output of $G_{2}$ are $U_{T}=\operatorname{Re}\left\{V_{T}\right\}$ and $Y_{T}=\operatorname{Re}\left\{G_{2}\left(e^{j \omega}\right) V_{T}\right\}$ where $V_{T}$ is the same as above. Figure 3 plots the vectors $V_{T}$ (red) and $G_{2}\left(e^{j \omega}\right) V_{T}$ (green) in the complex plane. The projection of these points onto the real axis corresponds with the inputoutput data $U_{T}$ and $Y_{T}$. Note that the green data has points of the form $(a \pm j b)$ for some $(a, b)$. Projecting these points to the real axis results in repeats in the entries of $Y_{T}$. As a result, the interpolation $\phi$ is multivalued with a stair-step shape as shown in Figure 4 


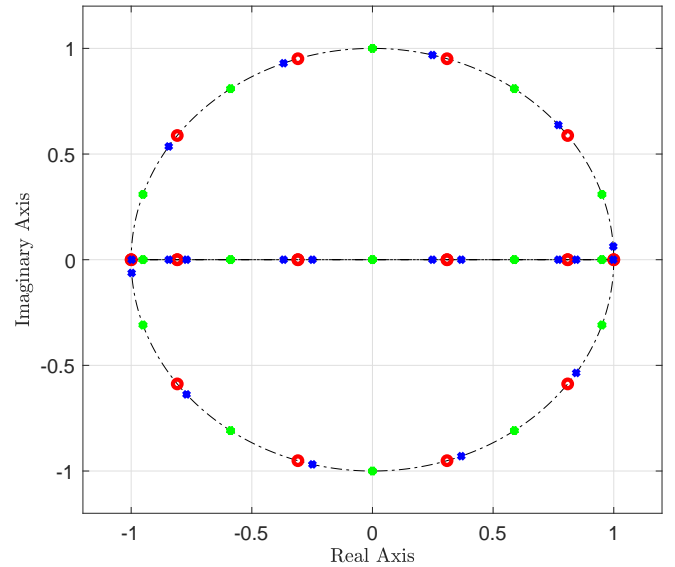

Fig. 3: Points $V_{T}, G_{1}\left(e^{j \omega}\right) V_{T}$, and $G_{2}\left(e^{j \omega}\right) V_{T}$ in the complex plane.

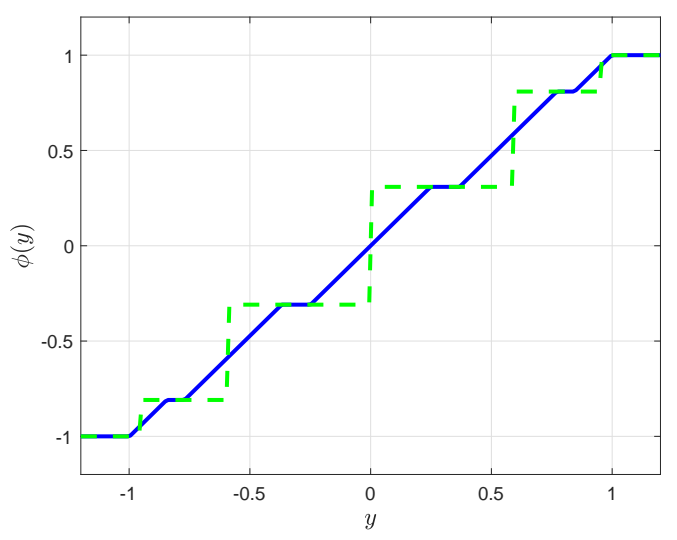

Fig. 4: Interpolated nonlinearities for $G_{1}$ (blue) and $G_{2}$ (green).

\section{B. Example with $\alpha$ even and $k=\infty$}

Let $\alpha=2, \beta=3$, hence $\omega=2 \pi / 3$. The periodic solutions have period $T=3$. Consider two different plants: e.g. $G_{1}\left(e^{j \omega}\right)=-e^{j \frac{\pi}{6}}$ and $G_{2}\left(e^{j \omega}\right)=-e^{j \frac{\pi}{3}}$. The input and outputs are $U_{T}=\operatorname{Re}\left\{V_{T}\right\}$ and $Y_{T}=\operatorname{Re}\left\{G_{i}\left(e^{j \omega}\right) V_{T}\right\}(i=1,2)$ where:

$$
\begin{aligned}
V_{T} & :=\left[\begin{array}{lll}
1 & e^{j \frac{2 \pi}{3}} & e^{-j \frac{2 \pi}{3}}
\end{array}\right]^{\top} \\
G_{1}\left(e^{j \omega}\right) V_{T} & =\left[\begin{array}{lll}
e^{-j \frac{5 \pi}{6}} & e^{-j \frac{\pi}{6}} & e^{j \frac{\pi}{2}}
\end{array}\right]^{\top} \\
G_{2}\left(e^{j \omega}\right) V_{T} & =\left[\begin{array}{lll}
e^{-j \frac{2 \pi}{3}} & 1 & e^{-j \frac{2 \pi}{3}}
\end{array}\right]^{\top} .
\end{aligned}
$$

In this example we illustrate the interpolated nonlinearities. If we consider the set $S_{0, \infty}$, we see that $G_{1}$ is not a limiting case since it has finite slope, whereas $G_{2}$ is a limiting case as it is multi-valued, see Figure 5. Moreover, the interpolated nonlinearity is non-odd and it requires a shifting as explained in the proof to obtain $\phi(0)=0$. This shifting procedure is demonstrainted in the following section.

On the other hand, if we reduce our attention to $S_{0, \infty}^{\text {odd }}$, by ensuring oddness, $G_{1}$ becomes a limiting case as it is multi-valued, see Figure 6 In addition, the required odd nonlinearity for $G_{2}$ is not monotone. However, it does not contradict Theorem 2, as condition 15 is not satisfied for $G_{2}$.

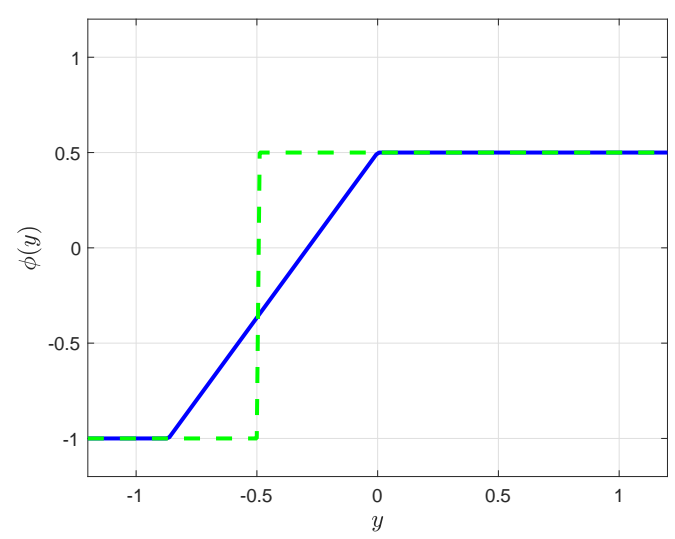

Fig. 5: Interpolated nonlinearities required for the Lurye system to have a periodic behaviour for $G_{1}$ and $G_{2}$. As the pair of points are nonodd, the interpolated nonlinearities are nonodd and do not cross the origin.

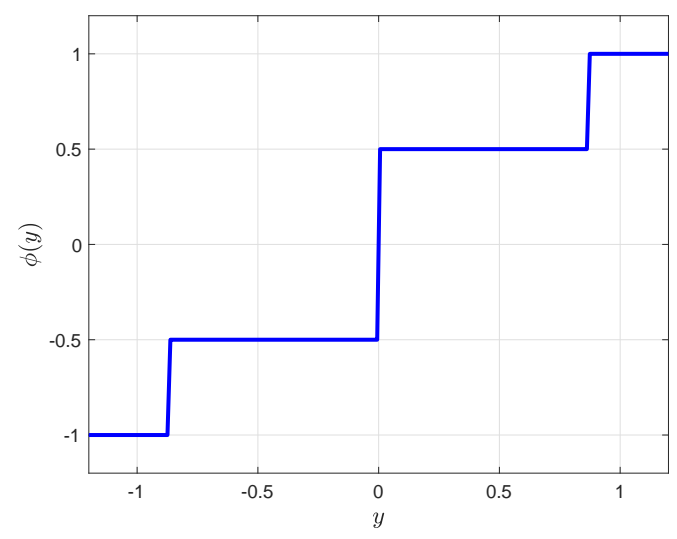

Fig. 6: Interpolated nonlinearities required for the Lurye system to have a periodic behaviour for $G_{1}$. In this case, $G_{1}$ is multi-values and we cannot ensure the existence of a nonlinearity within $S_{0, \infty}^{\text {odd }}$ resulting in a periodic behaviour. For $G_{2}$, the interpolated nonlinearity does not belong to $S_{0, \infty}^{\text {odd }}$.

\section{Examples with $k<\infty$}

Consider the following system:

$$
G(z)=\frac{z}{z^{2}-1.8 z+0.81}
$$

This plant has been used in [10], [11] as a second-order counterexample of the discrete-time Kalman Conjecture. The feedback interconnection of $G$ and a (linear) gain $k$ is stable if $0 \leq k<3.61$. A 4-periodic cycle was constructed for a slope-restricted nonlinearity with maximum slope $k=2.1$.

As mentioned in the introduction, Zames-Falb multipliers can be used to compute a lower bound on $k_{A S}$. Using the convex search in [7] yields multipliers that guarantee the stability for all $\phi \in S_{0, \underline{k}_{1}}$ with $\underline{k}_{1}=1.3028317$ and for all $\phi \in$ $S_{0, \underline{k}_{2}}^{\text {odd }}$ with $\underline{k}_{2}=1.3511322$. We use the results in this paper to construct destabilizing nonlinearities. This construction provides an upper bound $\bar{k} \geq k_{A S}$. For this plant the upper bounds are close to the Zames-Falb lower bounds and hence the conservatism in either bound is small. 


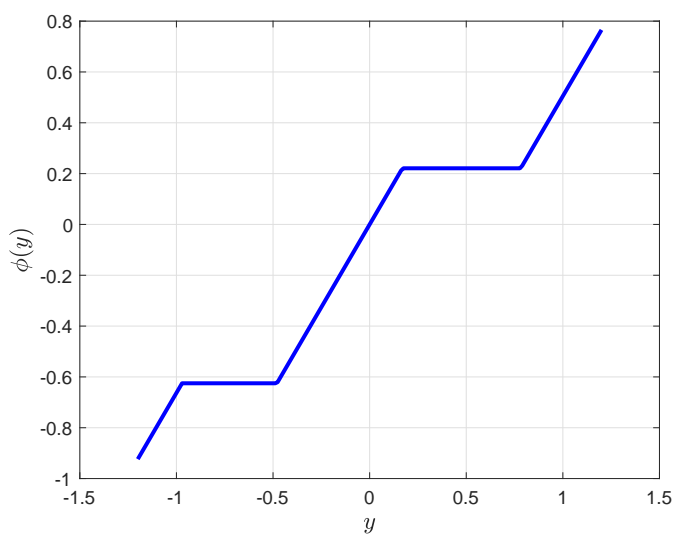

Fig. 7: Interpolated nonlinearity $\phi \in S_{0,1.3028373}$. The Lurye system with $\phi$ and $G$ in 19 exhibits a periodic behaviour.

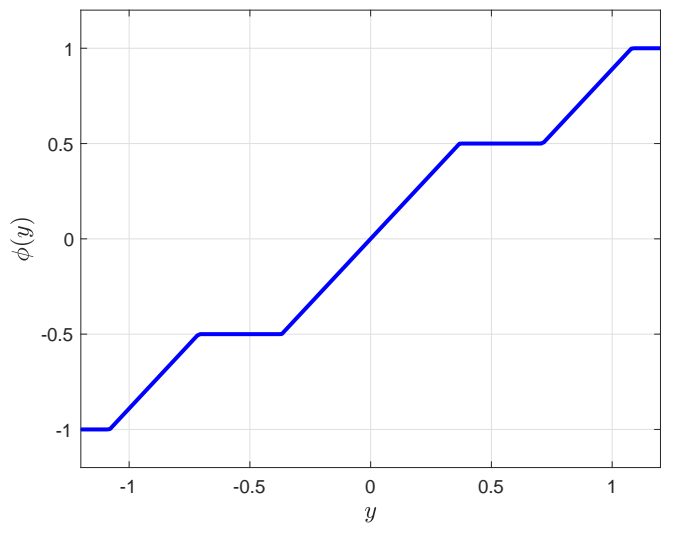

Fig. 8: Interpolated nonlinearity $\phi \in S_{0,1.3575410}^{\text {odd }}$ The Lurye system with $\phi$ and $G$ in 19 exhibits a periodic behaviour.

First consider the class of non-odd nonlinearities. Apply Proposition 1 using a large combination of values for $\alpha$ and $\beta$. We find that the minimum value of $\bar{k}$ is obtained for $\alpha=2$ and $\beta=7$. For these values, Proposition 1 ensures periodic behaviour for all $k \geq 1.3028373$. The required nonlinearity is depicted in Figure 7. To obtain this nonlinearity, we have to use Equation (14). For this particular plant, the DC gain of the loop transformed plant is $\tilde{G}(1)=100+1 / \bar{k} \simeq 100.7676$ and the shifting constant is $\xi=1.5985 \times 10^{-3}$.

Next consider the class of odd nonlinearities. Apply Proposition 2 for a large combination of values for $\alpha$ and $\beta$. We find that the minimum value of $\bar{k}^{\text {odd }}$ is obtained for $\alpha=1$ and $\beta=3$. Then, $\omega=\frac{\pi}{3}$ and $T=6$. For these values, Proposition 1 ensures periodic behaviour for all $k \geq$ 1.3575410. The required nonlinearity is depicted in Figure 8

\section{CONCLUSIONS}

This paper shows the connection between frequencydomain duality conditions for Zames-Falb multipliers developed in [9] and periodic behaviour of the Lurye system for slope-restricted nonlinearity. We develop an analytical construction for destabilizing nonlinearities. For all examples in [7], the construction yields systematic counterexamples of the discrete-time Kalman conjecture, and therefore less conservative upper bounds for absolute stability.

\section{REFERENCES}

[1] R. O'Shea, "An improved frequency time domain stability criterion for autonomous continuous systems," IEEE Transactions on Automatic Control, vol. 12, no. 6, pp. 725 - 731, December 1967.

[2] R. O'Shea and M. Younis, "A frequency-time domain stability criterion for sampled-data systems," IEEE Transactions on Automatic Control, vol. 12, no. 6, pp. 719-724, December 1967.

[3] G. Zames and P. L. Falb, "Stability conditions for systems with monotone and slope-restricted nonlinearities," SIAM J. Control, vol. 6, pp. 89-108, 1968.

[4] J. Willems and R. Brockett, "Some new rearrangement inequalities having application in stability analysis," IEEE Transactions on Automatic Control, vol. 13, no. 5, pp. 539-549, October 1968.

[5] J. C. Willems, The analysis of feedback systems, ser. Research monographs. Cambridge, Mass.: M.I.T. Press, 1971, no. 62.

[6] J. Carrasco, M. C. Turner, and W. P. Heath, "Zames-Falb multipliers for absolute stability: from O'Shea's contribution to convex searches," European Journal of Control, vol. 28, pp. 1-19, 2016.

[7] J. Carrasco, W. P. Heath, J. Zhang, N. S. Ahmad, and S. Wang, "Convex searches for discrete-time Zames-Falb multipliers," IEEE Transactions on Automatic Control, in press, 2020.

[8] S. Wang, J. Carrasco, and W. P. Heath, "Phase limitations of ZamesFalb multipliers," IEEE Transactions on Automatic Control, vol. 63, no. 4, pp. 947-959, April 2018.

[9] J. Zhang, J. Carrasco, and W. Heath, "Duality bounds for discrete-time Zames-Falb multipliers," 2020. [Online]. Available: https://arxiv.org/abs/2008.11975

[10] J. Carrasco, W. P. Heath, and M. de la Sen, "Second-order counterexample to the discrete-time Kalman conjecture," in European Control Conference, 2015, pp. 981-985.

[11] W. P. Heath, J. Carrasco, and M. de la Sen, "Second-order counterexamples to the discrete-time Kalman conjecture," Automatica, vol. 60, pp. $140-144,2015$.

[12] R. Fitts, "Two counterexamples to Aizerman's conjecture," IEEE Transactions on Automatic Control, vol. 11, no. 3, pp. 553-556, 1966.

[13] N. E. Barabanov, "On the Kalman problem," Siberian Mathematical Journal, vol. 29, no. 3, pp. 333-341, 1988.

[14] V. Bragin, V. Vagaitsev, N. Kuznetsov, and G. Leonov, "Algorithms for finding hidden oscillations in nonlinear systems. the Aizerman and Kalman conjectures and Chua's circuits," Journal of Computer and Systems Sciences International, vol. 50, no. 4, p. 511, 2011.

[15] G. A. Leonov and N. V. Kuznetsov, "Hidden attractors in dynamical systems. from hidden oscillations in Hilbert-Kolmogorov, Aizerman, and Kalman problems to hidden chaotic attractor in Chua circuits," Int. J. of Bifurcation and Chaos, vol. 23, no. 01, p. 1330002, 2013.

[16] T. Zvyagintseva, "On the Aizerman problem: Coefficient conditions for the existence of a four-period cycle in a second-order discretetime system," Vestnik St. Petersburg University, Mathematics, vol. 53, no. 1 , pp. 37-44, 2020 .

[17] — - "On the Aizerman problem: Coefficient conditions for the existence of three-and six-period cycles in a second-order discretetime system," Vestnik St. Petersburg University, Mathematics, vol. 53, pp. 206-213, 2020.

[18] B. Lee and P. Seiler, "Finite step performance of first-order methods using interpolation conditions without function evaluations," 2020. [Online]. Available: https://arxiv.org/abs/2005.01825

[19] C. A. Desoer and M. Vidyasagar, Feedback systems: input-output properties. SIAM, 2009.

[20] D. Lambert, J.-P. Crouzeix, V. Nguyen, and J. Strodiot, "Finite convex integration," J. of Convex Analysis, vol. 11, no. 1, pp. 131-146, 2004.

[21] A. Taylor, J. Hendrickx, and F. Glineur, "Smooth strongly convex interpolation and exact worst-case performance of first-order methods," Mathematical Programming, vol. 161, pp. 307-345, 2017.

[22] A. Taylor, "Convex interpolation and performance estimation of firstorder methods for convex optimization," Ph.D. dissertation, Universite Catholique de Louvain, 2017.

[23] A. Gelb and W. E. Vander Velde, Multiple-input describing functions and nonlinear system design. McGraw-Hill, New York, 1968.

[24] G. Golub and C. Van Loan, Matrix Computations, ser. Johns Hopkins Studies in the Mathematical Sciences. Johns Hopkins University Press, 2013.

[25] R. E. Kalman, "Physical and mathematical mechanisms of instability in nonlinear automatic control systems," Transactions of ASME, vol. 79, pp. 553-566, 1957. 